\title{
Left Inguinal Mass Presenting as an Incarcerated Left Inguinal Hernia in a Woman
}

\author{
Bin Li, MD, Yi-Ping Zhu, MD, Di-Yu Huang, MD, Neng-Yun Zhang, MD, Xian-Fa Wang, MD \\ Department of General Surgery \& Institute of Micro-invasive Surgery, Sir Run Run Shaw Hospital, Zhejiang University \\ School of Medicine, Hangzhou, China (all authors).
}

\begin{abstract}
Primary round ligament leiomyoma is uncommon, and an inguinal hernia due to primary round ligament leiomyoma is extremely rare. We present the case of a woman who underwent successful laparoscopic radical excision of this lesion. The patient was admitted with an asymptomatic mass in the left inguinal region suggesting an inguinal hernia. After physical, laboratory, and imaging examinations, a laparoscopic radical excision was performed. Histopathologic studies confirmed the diagnosis of primary round ligament leiomyoma.
\end{abstract}

Key Words: Hernia, Oncologic surgery, General laparoscopy.

Citation Li B, Zhu Y-P, Huang D-Y, Zhang N-Y, Wang X-F. Left inguinal mass presenting as an incarcerated left inguinal hernia in a woman. CRSLS e2014.11190. DOI 10.4293/CRSLS.2014.11190.

Copyright (C) 2014 SLS This is an open-access article distributed under the terms of the Creative Commons Attribution-Noncommercial-ShareAlike 3.0 Unported license, which permits unrestricted noncommercial use, distribution, and reproduction in any medium, provided the original author and source are credited.

The authors thank Prof. Iain C. Bruce (Zhejiang University School of Medicine) for improving the use of English in the manuscript.

Address correspondence to: Xian-Fa Wang, MD, Department of General Surgery \& Institute of Micro-invasive Surgery, Sir Run Run Shaw Hospital, Zhejiang University School of Medicine, East Qing-Chun Road No. 3, Hangzhou, China, 310016. Telephone: 86-571-86006445, Fax: 86-571-86044817, E-mail: zijinyanjing@zju.edu.cn

\section{INTRODUCTION}

Primary leiomyoma of the round ligament is uncommon, occurring predominantly in premenopausal middle-aged women. ${ }^{1}$ An inguinal hernia due to this type of leiomyoma is extremely rare and seldom seen in the literature.2,3 The exact pathogenesis is still unclear, and treatment is variable. We report a successful laparoscopic radical excision of this lesion.

\section{CASE REPORT}

A 39-year-old Chinese woman, gravida 2 para 1, was admitted with an asymptomatic mass in the left inguinal region, which had rapidly grown over a period of 5 months. Physical examination showed a firm, round, painless mass forming a left inguinal hernia with a diameter of $2.5 \mathrm{~cm}$; the mass was partly reducible when she was lying down. No gastrointestinal obstructive symptoms were found. She had no history of surgery, uterine leiomyoma, or long-term estrogen use. The laboratory results, including complete blood count, hepatorenal function test, urine analysis, and tumor markers, were all normal except a slightly high leukocyte count. The chest radiograph was also normal. Abdominal and transvaginal sonography showed a $9.31 \times 3.78$-cm tumor without ascites in the left abdominal cavity. A contrast-enhanced abdominal pelvic computed tomography (CT) scan showed a well-circumscribed $3.4 \times 4.7 \times 10$-cm heterogeneous mass connecting to a thickened left round ligament of the uterus (Figures 1 and 2). The diagnosis was a left inguinal hernia due to primary leiomyoma or leiomyosarcoma of the round ligament.

The patient underwent laparoscopic resection of the mass. The intraoperative findings showed a $6 \times 7 \times 10-\mathrm{cm}$ extraperitoneal mass in the left round ligament and partially extending into the left inguinal canal, forming an indirect inguinal hernia (Figure 3). No additional masses were noted, and the other organs in the abdomen were all normal. The round ligament that covered the mass was incised and dissected carefully. After total extrusion, the mass was cut into several fragments and completely removed from the round ligament. The ligament was closed by suture lines. Lymphadenectomy, which may lead to extra injuries, was not performed because the mass was well encapsulated and intraoperative frozen-section examination showed a benign tumor (leiomyoma). Macroscopically, the cut surface was white and whorled. Microscopic examination showed a bundled arrangement of 


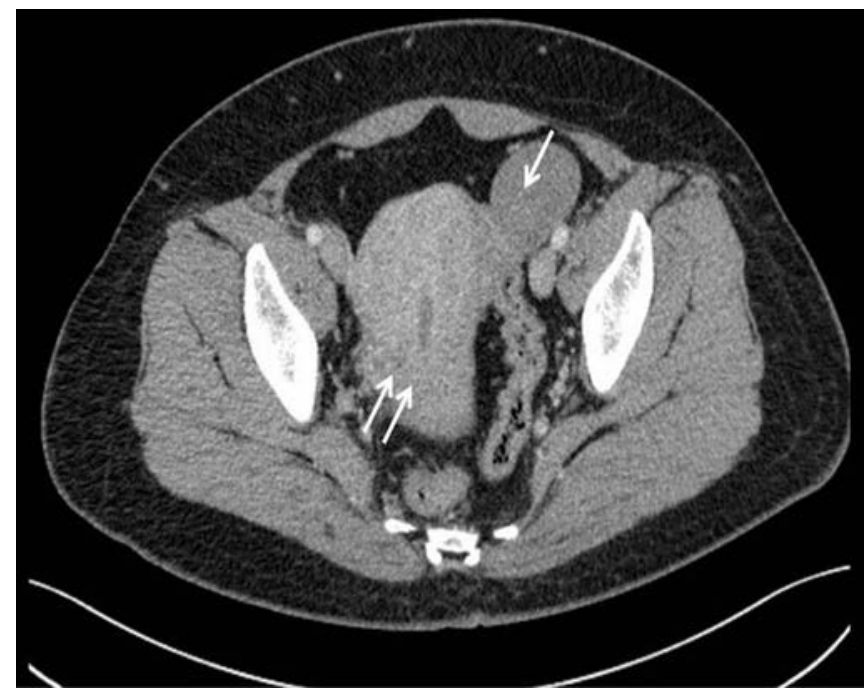

Figure 1. Contrast-enhanced abdominal pelvic CT scan showing heterogeneous mass in left round ligament (single arrow). The double arrows show the uterus.

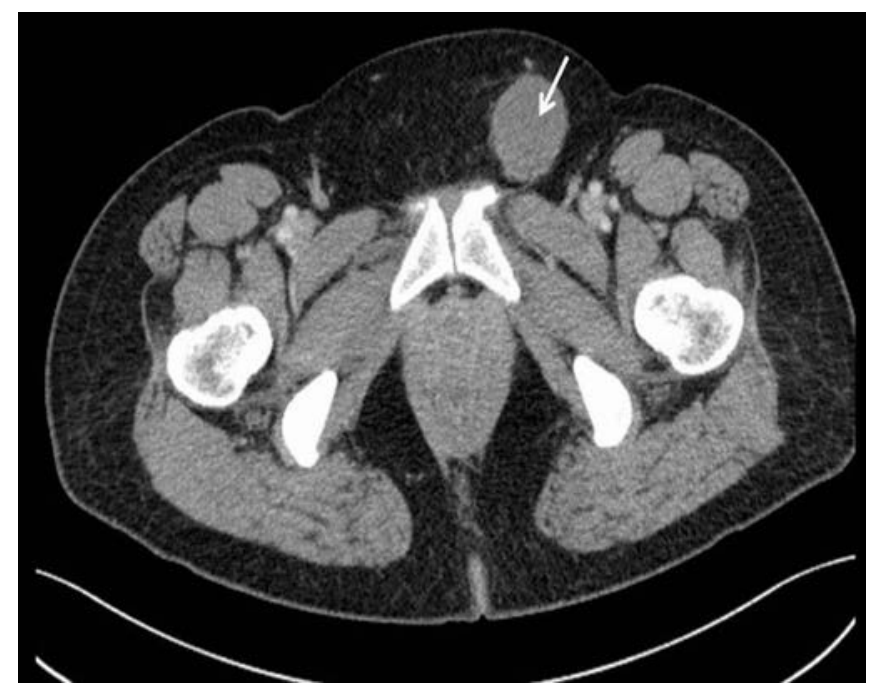

Figure 2. Contrast-enhanced abdominal pelvic CT scan showing heterogeneous mass in left inguinal region forming an inguinal hernia (arrow).

spindle-shaped cells with hyaline degeneration in some areas. Neither mitotic figures nor atypical nuclei were seen, which indicates leiomyoma (Figure 4). The postoperative course was uneventful, and the patient was discharged on the third day after surgery. To prevent recurrence, we suggested that the patient undergo abdominal and transvaginal sonography every 3 months. Six months of follow-up showed no recurrence of the hernia or tumor.

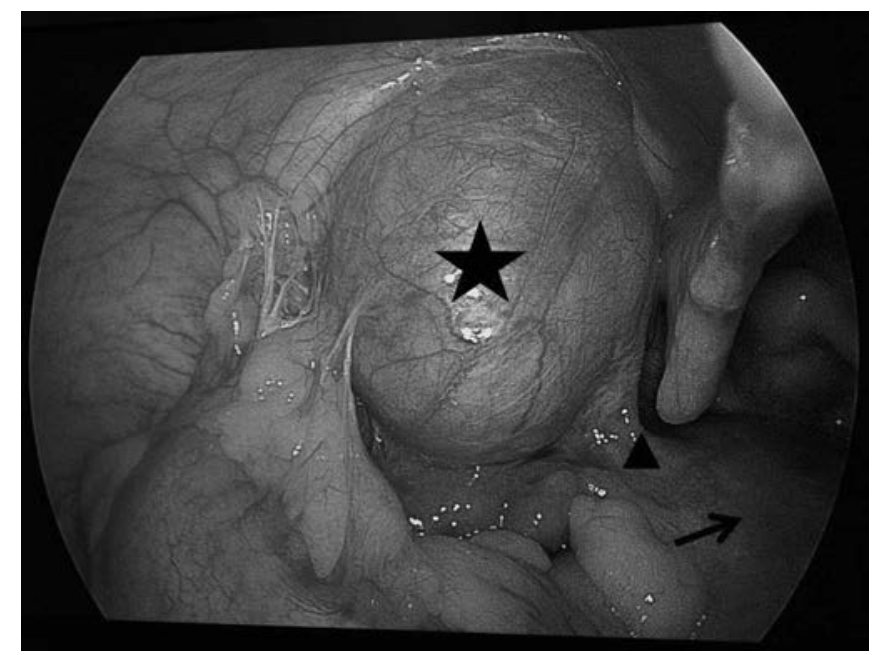

Figure 3. Laparoscopic excision of primary round ligament leiomyoma. The star indicates the mass in the round ligament forming an inguinal hernia. The arrowhead shows part of the round ligament. The arrow shows the uterus.

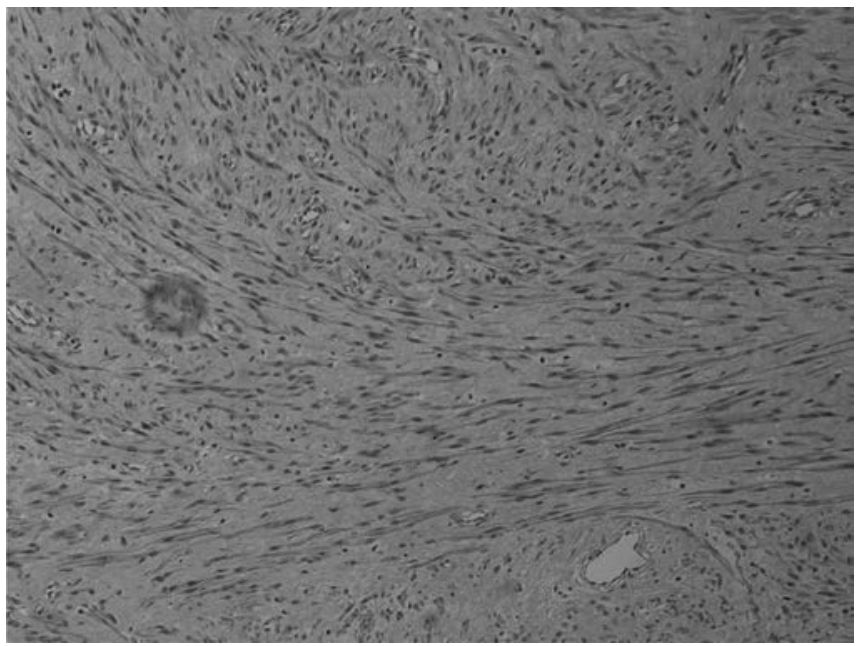

Figure 4. Microscopic view of leiomyoma in left round ligament (hematoxylin-eosin stain, original magnification $\times 100$ ). The tumor cells were spindle shaped, and blood vessels were noted. Mitoses, cellular irregularity, and nuclear pleomorphism were not evident.

\section{DISCUSSION}

Compared with the uterus, primary tumors of the round ligament are rare. Leiomyomas, which comprise one-third of round ligament tumors, are the most common lesion, followed by endometriomas and mesothelial cysts. ${ }^{2,4,5}$ Five consecutive series spanning the mid 19th century to 1962 documented a total of 110 cases, ${ }^{4,6}$ but an inguinal hernia due to primary round ligament leiomyoma is ex- 
tremely rare. ${ }^{2,3}$ Primary leiomyoma of the round ligament is reported more commonly on the right side with no satisfactory explanation, ${ }^{2,4}$ which makes our left-sided case even more rare. The average size of the mass ranges from 3 to $5 \mathrm{~cm},{ }^{2}$ whereas the lesion reported in our patient was relatively larger. On clinical presentation, patients are usually asymptomatic or show only a palpable mass in the abdomen, depending on which part of the ligament is involved. Intra-abdominal lesions are usually asymptomatic or show palpable masses. In contrast, extraperitoneal lesions present larger masses or even inguinal hernias, as in our case, which may lead to misdiagnosis and inappropriate treatment. ${ }^{2,7}$

Abdominal and transvaginal sonography is a more convenient diagnostic method that can detect masses in the abdomen, but it cannot distinguish their origin. Contrast-enhanced abdominal pelvic CT is more valuable in evaluating the extent and size of the lesion. The typical image shows a slightly heterogeneous mass with or without calcification points. ${ }^{2,7}$ Magnetic resonance imaging shows a mass of isosignal intensity on T1 weighting and a heterogeneous and high-intensity signal in some regions because of cystic or hyaline degeneration on T2 weighting. ${ }^{3}$

It is not difficult to recognize an inguinal hernia caused by a primary round ligament tumor on an imaging examination. However, since round ligament tumors are seldom seen, they may be confused with pedunculated uterine leiomyomas or ovarian tumors. ${ }^{7}$ The differential diagnosis, including ovarian or uterine masses, round ligament leiomyosarcoma, and other tumors originating from the round ligament, is difficult to make. A preoperative enhanced CT scan is helpful in distinguishing round ligament leiomyoma from mesenteric and endometrial cysts because of its solid enhanced nature. ${ }^{2}$ Primary round ligament leiomyosarcoma can be confirmed by pathologic examination with far more atypical cells, mitotic figures, and necrosis. Malignant perivascular epithelioid cell tumor and solitary fibrous tumor of the round ligament, which are reported to mimic leiomyoma on CT or magnetic resonance imaging, must be assessed by immunohistochemistry. $5,7,8$
Laparoscopic surgical procedures have developed rapidly in the past 20 years and are widely accepted because they are minimally invasive and yield a rapid recovery. A primary leiomyoma of the round ligament reported more than 10 years earlier was excised by laparotomy. ${ }^{2,4}$ Our experience shows that laparoscopic radical excision of inguinal hernias due to primary round ligament leiomyoma is feasible and safe. Because the growth of leiomyomas is estrogen dependent, the use of antiestrogens such as the gonadotropin-releasing hormone analogues or tamoxifen has been proposed, but their efficacy is still controversial. Recurrence after complete excision has not been reported, and long-term follow-up is needed to reaffirm this favorable prognosis.

\section{References:}

1. Lösch A, Haider-Angeler MG, Kainz C, et al. Leiomyoma of the round ligament in a postmenopausal woman. Maturitas. 1999;31:133-135.

2. Warshauer DM, Mandel SR. Leiomyoma of the extraperitoneal round ligament: CT demonstration. Clin Imaging. 1999;23: 375-376.

3. Rhee CS, Kim JS, Woo SK, et al. MRI of round ligament leiomyoma associated with Mayer-Rokitansky-Kuster-Hauser syndrome. Abdom Imaging. 1999;24:202-204.

4. Wilson SK, Smith RS, Sarratt MH. Large leiomyomas of the round ligament. South Med J. 1972;65:604.

5. Phongnarisorn C, Khunamornpong S, Pattamapaspong N, et al. Laparoscopic radical excision of primary round ligament perivascular epithelioid cell tumor mimicking leiomyoma. $J$ Minim Invasive Gynecol. 2009;16:626-629.

6. Kirkham JC, Nero CJ, Tambouret RH, et al. Leiomyoma and leiomyosarcoma arising from the round ligament of the uterus. J Am Coll Surg. 2008;207:452.

7. Pattamapaspong N, Khunamornpong S, Phongnarisorn C, et al. Malignant perivascular epithelioid cell tumour of the round ligament mimics leiomyoma on computed tomography. Singapore Med J. 2009;50:239-242.

8. Porter E, McGregor A, Brown L, et al. Solitary fibrous tumour of round ligament mimicking a leiomyoma. J Obstet Gynaecol. 2008;28:463-464. 